\title{
Endodontic treatment-related antibiotic prescribing patterns of South African oral health professionals
}

\author{
R. Lalloo, G. Solanki, K. Ramphoma and N. G. Myburgh
}

\begin{abstract}
Aim To assess the antibiotic prescribing patterns of South African dentists for patients undergoing endodontic treatment.

Methodology This study used data from 2013 health insurance claims submitted by South African oral health professionals to determine the antibiotic prescribing patterns related to endodontic treatment. A logistic regression model was used to test the fully adjusted statistical significance of the association between the exploratory variables (gender, age group, event type, abscess treatment, chronic health) and the dependent variable (antibiotic prescription). Odds ratios with 95\% confidence intervals (CI) are reported, and a 95\% CI excluding 1 was considered statistically significant.

Results Almost $10 \%$ of endodontic treatments were prescribed an antibiotic. There were no significant differences in prescribing patterns by gender, age and chronic health status. Prescriptions were more common at the preparatory stage (9.4\%) of root canal treatment compared to the therapy (4.7\%) and canal filling (2\%) stages. Patients who received apical surgery $(\mathrm{OR}=2.28$; 95\% CI 1.38-3.76) and treatment of an abscess (OR $=2.57 ; 95 \% 1.82-3.63)$ had a significantly increased odds of being prescribed an antibiotic. Almost three-quarters of prescriptions were for narrow spectrum antibiotics.

Conclusion The frequency of antibiotic prescribing by South African dental practitioners for patients undergoing endodontic treatment is relatively low and predominantly involved narrow spectrum antibiotics. It, however, remains important that antibiotics are only prescribed when clinically essential, such as when there are obvious systemic effects. These include fever above 37 degrees, malaise, lymphadenopathy, trismus, increase swelling, cellulitis, osteomyelitis and persistent infection. The wider dissemination and adherence to clear evidence-based prescribing guidelines for antibiotics in this clinical area are important.
\end{abstract}

\section{Introduction}

Antimicrobials are used extensively by healthcare professionals to treat or prevent bacterial, parasitic, viral and fungal infections. There is now an increasing global threat to the effectiveness of these agents from resistant organisms. The Lancet Infectious 
Disease Commission published a series of papers on antibiotic resistance to encourage more prudent use (Howard et al. 2013, The Lancet Infectious Diseases 2013).

In oral health, antibiotics are used to both treat oral infections and prevent them before, during or after dental treatment. Antibiotics are mainly used to treat severe periodontal infections and to prevent or treat surgery-related complications, such as following tooth extractions, endodontic therapy, trauma surgery, orthognathic surgery and implant placement (Seymour 2013, Oberoi et al. 2015, Walton 2016).

Root canal treatment is the removal of the bacteria and their by-products by thorough debridement of the root canal system, to reduce infection and curb inflammation (American Association of Endodontists 2006). In most cases, the patient's condition improves once the source of infection is eliminated. Antibiotic justification for dental infections requires that the infection be persistent or systemic in nature. Pain alone or localized swelling is not an indication for antibiotic treatment (European Society of Endodontology 2006). In an otherwise healthy patient with a small localized swelling, antibiotics are not indicated unless the patient also has signs and symptoms of infection and its spread (Henry et al. 2001). These may include fever above 37 degrees, malaise, lymphadenopathy, trismus, increase swelling, cellulitis, osteomyelitis and persistent infection. Conditions not requiring the use of antibiotics include pain without signs and symptoms of infection, teeth with necrotic pulps and radiolucency, teeth with a sinus tract and localized fluctuant swellings (American Association of Endodontists 2006).

The American Association of Endodontics has outlined that the responsible use of antibiotics in endodontic treatment should include narrow spectrum antibiotics as the first choice for prescription because the broad-spectrum antibiotics tend to produce more changes in the normal gastrointestinal tract, which may contribute to organism resistance. The recommended antibiotics include penicillin VK, amoxicillin with clavulanate, clindamycin, metronidazole, erythromycin, clarithromycin, cephalosporins and doxycycline (American Association of Endodontists 2006).

Currently, there are few studies on the use of antibiotics in patients undergoing endodontic therapy. A study of antibiotic prescribing by oral surgeons for endodontic infections reported that 95\% selected amoxicillin as their first choice (Segura-Egea et al. 2010). This was used alone in $34 \%$ of responses and associated with clavulanate in $61 \%$. The same study found that a significant majority would prescribe antibiotics for irreversible pulpitis (86\%), for necrotic pulps, acute apical periodontitis and no swelling (71\%) and for necrotic pulps with chronic apical periodontitis and a sinus tract (60\%). A survey of endodontists found that a minority would prescribe antibiotics for cases of irreversible pulpitis (16.76\%) and necrotic pulps with chronic apical periodontitis and a sinus tract (12\%) (Yingling et al. 2002). For a necrotic pulp, acute apical periodontitis and no swelling, more than half would prescribe an antibiotic (53.93\%). Nabavizadeh et al. (2011) reported that only 29\% of their 
participants demonstrated a full knowledge regarding the correct use of antibiotics in pulpal and periapical disease. They concluded that there was generally poor knowledge of valid indications for prescription, type and dosage of antibiotics. A study found that in $4.2 \%$ of all patient encounters, antibiotics were prescribed (Mainjot et al. 2009). Of these, broad-spectrum antibiotics were most commonly prescribed (82\%), and these were prescribed in the absence of fever (92.2\%) and without any other local treatment (54.2\%). Antibiotics were prescribed to $63.3 \%$ of patients with a periapical abscess and $4.3 \%$ of patients with pulpitis.

There does not appear to be a consistent pattern in prescribing antibiotics for endodontic infections and during endodontic treatment. Faced with the serious threat of antibiotic resistance and the costs of inappropriate antibiotic use, it is important to better understand the patterns of antibiotic prescribing related to endodontic treatment by oral health professionals. This is essential if the appropriate use of antibiotics in endodontics is to be achieved. This study analyses the antibiotic prescribing patterns related to endodontic treatment by South African oral health professionals to assess the alignment of these prescribing practices with the evidence available.

\section{Materials and methods}

There were approximately 8.78 million lives covered by private health insurance (medical) schemes in South Africa in 2013 (Council for Medical Schemes 2014). For this study, the 2013 claims for members of 11 health insurance schemes comprising 1.04 million lives (11.8\% of the insured population) were analysed. The data were obtained from the data warehouse of Towers Watson, a private firm providing consulting and actuarial services to South African health insurance schemes.

The analysis for this study required the data to be coded so that it would be possible to (i) identify events where members underwent endodontic treatment by dentists ('endodontic event'), (ii) categorize each event into an event type taking into account the type of endodontic treatment being provided, (iii) establish the demographic profile of the member being treated (age, gender, chronic health status) and (iv) determine for each endodontic event whether an antibiotic was prescribed or not.

For each service rendered in the treatment of members, the health provider submits a claim to the insurance scheme. Each submitted claim has at least the following information: (i) a unique identifier for the patient being treated, (ii) the date on which the service was rendered, (iii) a unique identifier for the provider providing the service, (iv) a code and description for each service rendered/item dispensed, (v) the ICD_10 code for the condition being treated, (vi) a NAPPI code (a unique identifier) for any surgical, medical or consumable item dispensed and (vii) the monetary amount claimed. 
For this study, all claims submitted for the endodontic treatment of a patient by a general dental practitioner and an antibiotic claim by a pharmacist on the same date were defined as an 'event'. Visits to a general dental practitioner where the submitted claims included endodontic treatment-related codes as listed in Table 1 were deemed to be an 'endodontic' event, and depending on the type of codes submitted, each event was categorized into the following types: (i) pulp treatment, (ii) root canal preparatory visit, (iii) root canal therapy, (iv) root canal obturation, (v) apical surgery, (vi) other and (vii) combinations of above treatments on the same visit. A summary of codes used to classify endodontic event types is presented in Table 1.

An event was considered to be positive for antibiotics prescribing if there was a claim related to the event for any of the following categories of antibiotics: beta-lactam antibiotics, cephalosporins, erythromycin and other macrolides, other beta-lactam antibiotics, penicillins, quinolones, sulphonamides and combinations and tetracyclines. An event was considered to be positive for the treatment of abscesses if claims for the event included claim codes 8731 (incision and drainage of abscess intra-oral), 9011 (incision and drainage of abscess - intra-oral - pyogenic) or 9013 (incision and drainage of abscess - extra-oral - pyogenic).

To assess the factors influencing antibiotic prescribing patterns in relation to endodontic treatment, prescribing patterns were analysed by the seven endodontic treatment types described earlier and the treatment of abscesses, by gender, age group (5 years or less; young children and adults aged 6-35 years; adults from 36 to 65 years and older adults 66+ years) and chronic health status. Based on the available data, it was possible to identify whether a member was receiving ongoing medication for the treatment of certain medical conditions. Study members receiving regular ongoing medication for the treatment of Diabetes, Cardiovascular disease, HIV or Asthma were classified as having a chronic illness for the purposes of this study. 
Table 1 Endodontic treatment codes; their description and category type

\begin{tabular}{|c|c|c|}
\hline Code & Description & Endodontic event type \\
\hline 8301 & Pulp cap - direct & Pulp treatment \\
\hline 8303 & Pulp cap - indirect & Pulp treatment \\
\hline 8307 & Pulp amputation (pulpotomy) & Pulp treatment \\
\hline 8132 & Pulp removal (pulpectomy) & Pulp treatment \\
\hline 8332 & Root canal preparatory visit - single canal tooth & Root canal preparatory visit \\
\hline 8333 & Root canal preparatory visit - multi canal tooth & Root canal preparatory visit \\
\hline 8338 & Root canal therapy - anteriors and premolars - first canal & Root canal therapy \\
\hline 8329 & Root canal therapy - anteriors and premolars - each additional canal & Root canal therapy \\
\hline 8339 & Root canal therapy - posteriors - first canal & Root canal therapy \\
\hline 8340 & Root canal therapy - posteriors - each additional canal & Root canal therapy \\
\hline 8631 & Root canal therapy - first canal & Root canal therapy \\
\hline 8633 & Root canal therapy - each additional canal & Root canal therapy \\
\hline 8335 & Root canal obturation - anteriors and premolars - first canal & Root canal obturation \\
\hline 8328 & Root canal obturation - anteriors and premolars - each additional canal & Root canal obturation \\
\hline 8336 & Root canal obturation - posteriors - first canal & Root canal obturation \\
\hline 8337 & Root canal obturation - posteriors - each additional canal & Root canal obturation \\
\hline 8635 & Apexification/recalcification - per visit & Apical surgery \\
\hline 9015 & Apicectomy - anteriors (including retrograde filling) & Apical surgery \\
\hline 9016 & Apicectomy - posteriors (including retrograde filling) & Apical surgery \\
\hline 8334 & Re-preparation of previously obturated root canal & Other \\
\hline 8330 & Removal of root canal obstruction & Other \\
\hline 8136 & Access through a prosthetic crown or inlay to facilitate root canal treatment & Other \\
\hline 8640 & Removal of fractured post or instrument from root canal & Other \\
\hline \multirow[t]{2}{*}{8765} & Hemisection of a tooth, resection of a root or tunnel preparation (isolated procedure) & Other \\
\hline & Combination: events with codes from more than 1 of above categories & \\
\hline
\end{tabular}

\section{Statistical analysis}

A logistic regression model was used to test the fully adjusted statistical significance of the association between the exploratory variables (gender, age group, event type, abscess treatment, chronic health) and the dependent variable (antibiotic prescription). Odds ratios with 95\% confidence intervals (CI) are reported, and a 95\% CI excluding 1 was considered statistically significant. SAS was used to summarize and consolidate the claims level data into 'event level' data (SAS Software for Windows Version 9.4.) Microsoft Excel was used to determine the frequency distributions of prescription patterns together with the odds ratios by the endodontic events categories and other variables.

\section{Results}

Ethical approval for the study was granted by the University of Western Cape Senate Research Committee, Project Registration Number: 15/3/24. Data for this study were accessed in terms of and under the conditions set out in the consulting agreement between Towers Watson and their client schemes. The data were analysed by Towers Watson internally and were not made available to any other third party. All findings are presented at an aggregate level, and at no point was confidential scheme or member information disclosed.

Of the 1.04 million lives analysed, $48.5 \%$ were female. By age group, $17.2 \%$ were under 10 years, $28.7 \%$ were $10-30$ years, $31.8 \%$ were $30-50$ years, 
$16.3 \%$ were $50-70$ years and $6 \%$ were older than 70 years. The average age of the members was 52 years. A quarter (24.8\%) of members experienced a chronic condition.

A total of 45305 endodontic treatment claims were found for this analysis. Of these 4480 (9.9\%) had an antibiotic claim from a pharmacist on the same day (Table 2). There were no differences in the distribution of antibiotics prescribed by gender, age group and chronic health status of the patient. Patients who received root canal treatment (preparatory visit, therapy and obturation) were at significantly decreased odds of being prescribed an antibiotic compared to those receiving pulp treatments. Patients who received apical surgery had a 2.28 (95\% CI $=1.38-3.76)$ increased odds of being prescribed an antibiotic compared to those receiving a pulp treatment. Patients who received treatment of an abscess by incision and drainage had a 2.57 (95\% CI $=1.82-3.63)$ increased odds of being prescribed an antibiotic compared to those not receiving this type of care. Almost a quarter (22\%) of patient who received treatment for an abscess were prescribed an antibiotic.

Forty-two per cent of the providers did not prescribe antibiotics for any of their treatment events. Twenty-one per cent of providers of providers prescribed antibiotics in $1-10 \%$ of events; $19 \%$ in $11-20 \%$ and $9 \%$ in $21-30 \%$. Eight per cent of providers prescribed an antibiotic in more than $30 \%$ of events.

Almost three-quarters (73.8\%) of prescriptions were for narrow spectrum antibiotics, such as penicillin, aminoglycosides and isoniazid (Table 3). Whilst the type of antibiotic prescribed did not differ markedly by the types of endodontic treatments, broad-spectrum antibiotics were prescribed slightly more often at the root canal obturation stage and for apical surgery. A combination of both narrow and broadspectrum antibiotics was seldom prescribed. 


\begin{tabular}{|c|c|c|c|}
\hline & $\begin{array}{l}\text { Number } \\
\text { of events }\end{array}$ & $\begin{array}{c}\text { Antibiotic } \\
\text { prescribed } \\
n(\%)\end{array}$ & OR $(95 \% \mathrm{Cl})$ \\
\hline Overall & 45305 & $4480(9.9)$ & \\
\hline \multicolumn{4}{|l|}{ Gender } \\
\hline Male & 27866 & $2735(9.8)$ & 1.00 \\
\hline Female & 17439 & $1745(10.0)$ & $1.02(0.96-1.09)$ \\
\hline \multicolumn{4}{|l|}{ Age group } \\
\hline$<10$ years & 391 & $40(10.2)$ & 1.00 \\
\hline $10-30$ years & 3408 & $334(9.8)$ & $0.95(0.67-1.34)$ \\
\hline $30-50$ years & 23975 & $2544(10.6)$ & $1.04(0.75-1.44)$ \\
\hline $50-70$ years & 11963 & $1093(9.1)$ & $0.88(0.63-1.23)$ \\
\hline$>70$ years & 5568 & $469(8.4)$ & $0.81(0.58-1.14)$ \\
\hline \multicolumn{4}{|l|}{ Event type } \\
\hline Pulp treatment & 18897 & $2865(15.2)$ & 1.00 \\
\hline $\begin{array}{l}\text { Root canal } \\
\text { preparatory visit }\end{array}$ & 4885 & $460(9.4)$ & $0.58(0.52-0.64)$ \\
\hline $\begin{array}{l}\text { Root canal } \\
\text { therapy }\end{array}$ & 12621 & $592(4.7)$ & $0.28(0.26-0.31)$ \\
\hline $\begin{array}{l}\text { Root canal } \\
\text { obturation }\end{array}$ & 5829 & $114(2.0)$ & $0.11(0.09-0.13)$ \\
\hline Apical surgery & 69 & $20(29.0)$ & $2.28(1.38-3.76)$ \\
\hline Other & 1157 & $162(14.0)$ & $0.91(0.77-1.08)$ \\
\hline $\begin{array}{l}\text { Combinations } \\
\text { of treatments }\end{array}$ & 1847 & $267(14.5)$ & $0.95(0.83-1.09)$ \\
\hline \multicolumn{4}{|l|}{ Abscess treatment } \\
\hline No & 45124 & $4440(9.8)$ & 1.00 \\
\hline Yes & 181 & $40(22.1)$ & $2.57(1.82-3.63)$ \\
\hline \multicolumn{4}{|l|}{ Chronic health } \\
\hline No & 135515 & 3075 (10.1) & 1.00 \\
\hline $\begin{array}{l}\text { Yes (Asthma/ } \\
\text { CVS/DM/HIV) }\end{array}$ & 31306 & $1405(9.5)$ & $0.94(0.88-1.00)$ \\
\hline
\end{tabular}

\section{Discussion}

The current study sought to investigate antibiotic prescription with endodontic treatment amongst South African dental practitioners. The 4480 (9.9\%) antibiotic claims from a pharmacist on the same day as the endodontic treatment represent a substantial proportion of the endodontic treatment taking place. Whilst direct comparisons to previous research are difficult, these findings are similar to those reported elsewhere. Nabavizadeh et al. (2011) and Gowri et al. (2015) respectively reported that $11 \%$ and $8.3 \%$ of their subjects were prescribed antibiotics after root canal therapy.

Similar to Segura-Egea et al. (2010) who found no significant variation in the management of endodontic infections by age and gender, this study found no difference in prescribing patterns by gender, age or chronic health status. SeguraEgea et al. (2010) reported the greatest number of antibiotic prescriptions was written for acute pulpits (80.6\%) and acute periapical abscess (74.2). For similar acute conditions, Iqbal (2015) reported that $77 \%$ of the dentists prescribed antibiotics for 
necrotic pulps with acute apical periodontitis and related features. In contrast, less than a quarter (22\%) of patients who received treatment for an abscess were prescribed an antibiotic in the current study.

In this study, almost three-quarters (73.8\%) of prescriptions were for narrow spectrum antibiotics, such as penicillin, aminoglycosides and isoniazid. This is in line with the American Association of Endodontics position that responsible use of antibiotics in endodontic treatment should include narrow spectrum antibiotics as the first choice of prescription, because broad-spectrum antibiotics tend to produce more changes in the normal gastrointestinal tract and lead to increased resistance (American Association of Endodontists 2006).

This South African study shows similar adherence to narrow spectrum prescription reported by others where narrow spectrum antibiotics were the first line of choice by most respondents (Segura-Egea et al. 2010, Iqbal 2015). Iqbal (2015) reported an amoxicillin and clavulanic acid combination was prescribed by $45.2 \%$, amoxicillin alone by $33.7 \%$ and an amoxicillin and metronidazole combination by $15 \%$. SeguraEgea et al. (2010) found amoxicillin in combination with calvulanic acid was prescribed by $61 \%$ of respondents and amoxicillin alone by $34 \%$. Peric et al. (2015) reported that the most common prescription was for a combination of amoxicillin and clavulanic acid (57.6\%), but these were for all clinical conditions. The first drug of choice for patients with an allergy to penicillins was clindamycin, in almost two thirds of respondents, followed by azithromycin and metronidazole-spiramycin.

A significant strength of the present study is the large number of events that were analysed and its ability to compare antibiotic prescribing patterns by stage of treatment and incision of an abscess. However, an important limitation was the inability of the data set to reveal pre-existing and existing clinical infection that may independently justify antibiotic use. It was also assumed that if the endodontic treatment and antibiotic events were on the same day, they were directly related. The authors are, however, confident that this would be true in almost all cases. Antibiotics related to the endodontic treatment administered on another day were not captured in this analysis. 
Table 3 Type of antibiotics prescribed by treatment type and stage

\begin{tabular}{|c|c|c|c|}
\hline & $\begin{array}{c}\text { Narrow } \\
\text { spectrum } \\
\text { only } \\
\text { n (\%) }\end{array}$ & $\begin{array}{l}\text { Broad } \\
\text { spectrum } \\
\text { only } \\
\text { n (\%) }\end{array}$ & $\begin{array}{c}\text { Narrow and } \\
\text { broad } \\
\text { spectrum } \\
\text { n }(\%)\end{array}$ \\
\hline Pulp treatment & $2154(75.2)$ & $698(24.4)$ & $13(0.5)$ \\
\hline $\begin{array}{l}\text { Root canal } \\
\text { preparatory visit }\end{array}$ & 329 (71.5) & $127(27.6)$ & $4(0.9)$ \\
\hline Root canal therapy & $460(77.7)$ & $127(21.5)$ & $5(0.8)$ \\
\hline $\begin{array}{l}\text { Root canal } \\
\text { obturation }\end{array}$ & $65(57.0)$ & 47 (41.2) & $2(1.8)$ \\
\hline Apical surgery & $12(60.0)$ & $7(35.0)$ & $1(5.0)$ \\
\hline Other & $103(63.6)$ & $58(35.8)$ & $1(0.6)$ \\
\hline $\begin{array}{l}\text { Combinations } \\
\text { of treatments }\end{array}$ & $185(69.3)$ & $79(29.6)$ & $3(1.1)$ \\
\hline Grand total & 3308 (73.8) & $1143(25.5)$ & $29(0.6)$ \\
\hline
\end{tabular}

South African dentists do not appear to be over-prescribing antibiotics for patients undergoing endodontic treatment, in relation to current norms as reflected in the literature, and predominantly narrow spectrum antibiotics are being prescribed. However, even at this relatively low rate of prescription, it is imperative that antibiotics are only prescribed when absolutely necessary to avoid the increasing global challenge of resistant organisms in a post-antibiotic era (Wolf 2015).

The antibiotic prescription rates reported in the literature and in this study are conservative but show wide variation in how and when antibiotics are used in relation to endodontic treatment in its different stages. This suggests that clinicians themselves do not appear to have a common understanding of exactly when antibiotics should be prescribed. The global dissemination of clear evidence-based guidelines for antibiotic prescription in this area of dental care seems to be important.

Such guidelines for antibiotic use in patients under-going endodontic treatment should at least discourage their use in an otherwise healthy patient with a small localized swelling. If the patient also has the established signs and symptoms of infection and the spread thereof, narrow spectrum antibiotics may be considered (American Association of Endodontists 2006, European Society of Endodontology 2006). 


\section{Conclusion}

Prescribing of antibiotics by South African dental practitioners for patients undergoing endodontic treatment is relatively low, and predominantly narrow spectrum antibiotics are prescribed. It, however, remains important that antibiotics are only prescribed when clinically essential, such as when there are obvious signs and symptoms of infection. These include fever above 37 degrees, malaise, lymphadenopathy and trismus, and increase swelling, cellulitis, osteomyelitis and persistent infection. The variation in prescribing practices across clinicians seen in this study strongly suggests that improved prescribing consistency is desirable. The wider dissemination and advocacy of clear evidence-based prescribing guidelines for antibiotics in endodontics may be a critical first step in the process.

\section{Funding}

No funding was received for this work.

\section{Conflict of interest}

The authors have stated explicitly that there are no conflict of interests in connection with this article. 


\section{References}

American Association of Endodontists (2006) Antibiotics and the Treatment of Endodontic Infections. Chicago, US: American Association of Endodontists.

Council for Medical Schemes (2014) Annual Report of the Council for Medical Schemes 2013/14. Council of Medical Schemes: Pretoria.

European Society of Endodontology (2006) Quality guidelines for endodontic treatment: consensus report of the European Society of Endodontology. International Endodontic Journal 39, 921-30.

Gowri S, Mehta D, Kannan S (2015) Antibiotic use in dentistry: a cross-sectional survey from a developing country. Journal of Orofacial Sciences 7, 90-4.

Henry M, Reader A, Beck M (2001) Effect of penicillin on postoperative endodontic pain and swelling in symptomatic necrotic teeth. Journal of Endodontics 27, 117-23.

Howard SJ, Catchpole M, Watson J, Davies SC (2013) Antibiotic resistance: global response needed. Lancet Infectious Diseases 13, 1001-3.

Iqbal A (2015) The attitudes of dentists towards the prescription of antibiotics during endodontic treatment in North of Saudi Arabia. Journal of Clinical and Diagnostic Research 9, ZC82-4.

Mainjot A, D’Hoore W, Vanheusden A, Van Nieuwenhuysen JP (2009) Antibiotic prescribing in dental practice in Belgium. International Endodontic Journal 42, 1112-7.

Nabavizadeh MR, Sahebi S, Nadian I (2011) Antibiotic prescription for endodontic treatment: general dentist knowledge + practice in Shiraz. Iranian Endodontic Journal 6, 54-9.

Oberoi SS, Dhingra C, Sharma G, Sardana D (2015) Antibiotics in dental practice: how justified are we? International Dental Journal 65, 4-10.

Peric M, Perkovic I, Romic M et al. (2015) The pattern of antibiotic prescribing by dental practitioners in Zagreb, Croatia. Central European Journal of Public Health 23, 10713.

SAS software for Windows Version 9.4. (2013) Cary, NC, 27513, USA: SAS Institute Inc.

Segura-Egea JJ, Velasco-Ortega E, Torres-Lagares D, Velasco-Ponferrada MC, MonsalveGuil L, Llamas-Carreras JM (2010) Pattern of antibiotic prescription in the management of endodontic infections amongst Spanish oral surgeons. International Endodontic Journal 43, 342-50.

Seymour RA (2013) Antibiotics in dentistry-an update. Dental Update 40, 319-22.

The Lancet Infectious Diseases (2013) Antibiotic resistance: long-term solutions require action now. Lancet Infectious Diseases 13, 995.

Walton RE (2016) Antibiotics: A Risky Prescription. Chicago, US: American Association of Endodontists.

Wolf J (2015) Antibiotic resistance threatens the efficacy of prophylaxis. Lancet Infectious Diseases 15, 1368-9.

Yingling NM, Byrne BE, Hartwell GR (2002) Antibiotic use by members of the American Association of Endodontists in the year 2000: report of a national survey. Journal of Endodontics 28, 396-404. 